\title{
Diverse phosphoregulatory mechanisms controlling cyclin-dependent kinase-activating kinases in Arabidopsis
}

\author{
Akie Shimotohno ${ }^{1,2}$, Ryoko Ohno ${ }^{1,3}$, Katerina Bisova ${ }^{1,4}$, Norihiro Sakaguchi ${ }^{1,3}$, Jirong Huang ${ }^{1,5}$, Csaba Koncz ${ }^{2}$, Hirofumi \\ Uchimiya $^{1}$ and Masaaki Umeda ${ }^{1,6, *}$ \\ ${ }^{1}$ Institute of Molecular and Cellular Biosciences, The University of Tokyo, Yayoi 1-1-1, Bunkyo-ku, Tokyo 113-0032, Japan, \\ ${ }^{2}$ Max-Planck Institute for Plant Breeding Research, Carl-von-Linné Weg 10, D-50829 Köln, Germany, \\ ${ }^{3}$ Department of Applied Biological Science, Faculty of Science and Technology, Tokyo University of Science, 2641 Yamazaki, \\ Noda-shi, Chiba 278-8510, Japan, \\ ${ }^{4}$ Institute of Microbiology, Academy of Sciences of the Czech Republic, Opatovicky mlyn, 379-81 Trebon, Czech Republic, \\ ${ }^{5}$ Shanghai Institute of Plant Physiology and Ecology, The Chinese Academy of Sciences, 300 Fenglin Road, Shanghai 200032, \\ China, and \\ ${ }^{6}$ Graduate School of Biological Sciences, Nara Institute of Science and Technology, Takayama 8916-5, Ikoma, Nara 630-0101, \\ Japan
}

Received 8 February 2006; revised 18 April 2006; accepted 11 May 2006.

*For correspondence (fax +81 74372 5599; e-mail: mumeda@bs.naist.jp).

\begin{abstract}
Summary
For the full activation of cyclin-dependent kinases (CDKs), not only cyclin binding but also phosphorylation of a threonine (Thr) residue within the T-loop is required. This phosphorylation is catalyzed by CDK-activating kinases (CAKs). In Arabidopsis three D-type CDK genes (CDKD;1-CDKD;3) encode vertebrate-type CAK orthologues, of which CDKD;2 exhibits high phosphorylation activity towards the carboxy-terminal domain (CTD) of the largest subunit of RNA polymerase II. Here, we show that CDKD;2 forms a stable complex with cyclin $\mathrm{H}$ and is downregulated by the phosphorylation of the ATP-binding site by WEE1 kinase. A knockout mutant of $C D K D ; 3$, which has a higher CDK kinase activity, displayed no defect in plant development. Instead, another type of CAK - CDKF; 1 - exhibited significant activity towards CDKA;1 in Arabidopsis root protoplasts, and the activity was dependent on the T-loop phosphorylation of CDKF;1. We propose that two distinct types of CAK, namely CDKF;1 and CDKD;2, play a major role in CDK and CTD phosphorylation, respectively, in Arabidopsis.
\end{abstract}

Keywords: cyclin-dependent kinase, CDK-activating kinase, cyclin H, WEE1, cell cycle, transcription.

Introduction

Progression through the eukaryotic cell cycle is controlled by the activity of cyclin-dependent serine (Ser)/threonine (Thr) protein kinases (CDKs). CDKs are activated by binding to specific cyclin partners, and the activity of cyclin-CDK complexes is further regulated by the synthesis and degradation of cyclin subunits, binding to inhibitory proteins, and the phosphorylation of CDKs themselves (Morgan, 1997). CDK phosphorylation is mediated by two groups of protein kinases: CDK-activating kinases (CAKs) and WEE1 kinases. CAKs are responsible for activating phosphorylation of conserved Thr residues within the T-loop (Kaldis, 1999). WEE1 kinases execute the inhibitory phosphorylation within the N-terminal ATP binding site and are counteracted by the action of Cdc25 phosphatases, which are essential for dephosphorylating and activating CDKs in order to trigger mitosis (Featherstone and Russell, 1991; Kumagai and Dunphy, 1991; Nurse, 1990).

The catalytic subunit of vertebrate CAK is termed CDK7/ $\mathrm{p} 40^{\mathrm{MO} 15}$, and its regulatory subunit is named cyclin $\mathrm{H}$ (Fisher and Morgan, 1994; Labbé et al., 1994; Mäkelä et al., 1994). In the presence of cyclin $\mathrm{H}, \mathrm{CDK} 7$ activity is significantly stimulated (Fisher and Morgan, 1994). A third subunit that stabilizes the cyclin H-CDK7 complex is a RING finger protein named MAT1 (Devault et al., 1995; Tassan et al., 1995). In addition to CDKs, vertebrate CAK phosphorylates the carboxy-terminal domain (CTD) of the largest subunit of RNA polymerase II. The trimeric CDK7-cyclin H-MAT1 complex has been identified in the general transcription factor TFIIH 
that is involved in the initiation and elongation of transcription (Schwartz et al., 2003; Serizawa et al., 1995; Shiekhattar et al., 1995). In fission yeast, CAK consists of Mcs6/Crk1/ Mop1, Mcs2 and Pmh1, which are closely related to CDK7, cyclin $\mathrm{H}$ and MAT1, respectively, and phosphorylates both Cdc2 and the CTD (Bamps et al., 2004; Buck et al., 1995; Damagnez et al., 1995).

In budding yeast, a monomeric CAK, namely Cak1p/Civ1p, has been shown to possess $\mathrm{Cdc} 28 \mathrm{p}$-activating kinase activity in vivo (Espinoza et al., 1996; Kaldis et al., 1996; Thuret et al., 1996). However, Cak1p has a very low sequence similarity to other CAKs and does not possess CTD kinase activity. The orthologue of CDK7 in budding yeast is Kin28p, which is associated with TFIIH and phosphorylates the CTD (Cismowski et al., 1995; Feaver et al., 1994, Feaver et al., 1997; Liu et al., 2004) but does not exhibit Cdc28p kinase activity. This indicates that CDK and CTD phosphorylations are controlled by distinct kinases in budding yeast. Kimmelman et al. (1999) have reported that Cak1p has another activity: to phosphorylate the T-loop of Kin28p and thereby stimulate its CTD kinase activity. This suggests that Cak1p is also involved in basal transcription.

Based on the primary structure, plant CDK-related proteins have been classified into six types, namely, CDKACDKF (Joubès et al., 2000; Vandepoele et al., 2002). Among them, CDKA is assumed to be an orthologue of $\mathrm{Cdc} 2 / \mathrm{Cdc} 28 \mathrm{p}$ and appears to function in both G1- to S-phase and G2- to Mphase progression (Hemerly et al., 1995). CDKD was assigned to the proteins that have a high similarity to vertebrate-type CAKs. The first plant CAK, namely Orysa;CDKD;1, was identified in rice plants (Hata, 1991) and was shown to be closely related to mammalian CDK7 in terms of enzyme activity (Yamaguchi et al., 1998, 2000). There are three CDKD genes in Arabidopsis: Arath;CDKD;1, Arath;CDKD;2 and Arath;CDKD;3 (originally named $C A K 3 A$ t, CAK4At and CAK2At, respectively) (Shimotohno et al., 2003; Umeda, 2002). Although both Arath;CDKD;2 and Arath;CDKD;3 (hereafter called CDKD;2 and CDKD;3, respectively) exhibited CDK and CTD kinase activities, Arath;CDKD;1 (hereafter called CDKD;1) showed neither CDK nor CTD kinase activity (Shimotohno et al., 2004). CDK2 kinase activity of CDKD;3 was extremely high compared with that of CDKD;2, whereas CDKD;2 had higher CTD kinase activity than CDKD;3 (Shimotohno et al., 2003). In insect cells a cyclin $\mathrm{H}$ homologue, namely Arath; $\mathrm{CycH} ; 1$ (hereafter called $\mathrm{CycH} ; 1)$, bound and activated CDKD;2 and CDKD;3, but not CDKD;1.

A distinct type of CAK is encoded on the CDKF gene. In Arabidopsis, Arath;CDKF;1 (originally designated as $C A$ $K 1 A t$ ) has been isolated as a suppressor of the CAK mutation in budding yeast (Umeda et al., 1998). The amino acid sequence is related to those of vertebrate-type CAKs, but similarities are restricted to the conserved kinase domains. Arath;CDKF;1 (hereafter called CDKF;1) phosphorylated and activated human CDK2 in vitro, but did not phosphorylate the CTD and was unable to interact with $\mathrm{CycH} ; 1$ (Shimotohno et al., 2004; Umeda et al., 1998). Recently, we revealed that $\mathrm{CDKF} ; 1$ phosphorylates the $\mathrm{T}$-loop of CDKD;2 and CDKD;3, and activates the CTD kinase activity of CDKD;2. Therefore, CDKF; 1 functions as a CAK-activating kinase as well as a CAK (Shimotohno et al., 2004; Umeda et al., 2005). Here, we show that CDKF; 1 exerted a high CAK activity in the absence of $\mathrm{CycH} ; 1$ and required T-loop phosphorylation for the activity. In contrast, CDKD;2 was tightly associated with $\mathrm{CycH} ; 1$ to display the CTD kinase activity, whereas it was negatively regulated via phosphorylation of the ATP binding site by the WEE1 kinase. We propose a model of CDK and CTD phosphorylation by distinct CAKs in Arabidopsis.

\section{Results}

\section{CycH;1 forms active kinase complexes with CDKDs} in plant cells

We have recently reported that Arabidopsis CDKD;2 and CDKD;3 interacted with $\mathrm{CycH;1}$ in yeast and insect cells; however, their interactions in plant cells remained unknown (Shimotohno et al., 2004). To identify $\mathrm{CycH} ; 1-\mathrm{CDKD}$ complexes, we prepared a specific antibody against histidine (His)-tagged $\mathrm{CycH}$;1. Immunoblot analyses performed using an Arabidopsis crude extract showed that three bands of 37, 39 and $40 \mathrm{kDa}$ were detected (Figure 1a). Depletion of the antibody from the antiserum by incubation with nickelnitriloacetic acid (Ni-NTA) agarose carrying $\mathrm{His}-\mathrm{CycH} ; 1$ resulted in the disappearance of all bands on the blots (Figure 1a). $\mathrm{CycH} ; 1$ produced in yeast cells showed the same mobility on SDS-PAGE as the $37-\mathrm{kDa}$ protein (Figure 1a), suggesting that it might represent the intrinsic $\mathrm{CycH} ; 1$. In plants, specific cross-reactions were observed in roots but were barely detected in shoots (Figure 1a).

The immunoprecipitation of an Arabidopsis crude extract with the anti- $\mathrm{CycH} ; 1$ antibody recovered all three proteins of 37,39 and $40 \mathrm{kDa}$; however, the 37-kDa protein was the most efficiently precipitated (Figure 1b). The same immunoprecipitates contained CDKD;2 but not CDKF;1 (Figure 1b). $\mathrm{CDKD} ; 3$ in the $\mathrm{CycH} ; 1$ immunoprecipitates was faintly detectable. Immunoprecipitation with the anti-CDKD antibodies showed that $\mathrm{CycH} ; 1$ was coprecipitated with $\mathrm{CDKD} ; 2$, but less efficiently with CDKD;3 (Figure 1b). These results indicate that at least $37-\mathrm{kDa} \mathrm{CycH} ; 1$ forms a stable complex with CDKD;2 in plant cells.

In the Arabidopsis cell culture, CDKD;2 forms a major complex with a molecular mass of $\sim 200 \mathrm{kDa}$, whereas CDKD;3 is included in two complexes of $\sim 130$ and $\sim 700 \mathrm{kDa}$, respectively (Shimotohno et al., 2004; Figure 1c). Here, we fractionated the total protein by Sephacryl S300 gel exclusion chromatography and immunoblotted each fraction with the anti-CycH;1 antibody. The 37-kDa protein was 
(a)
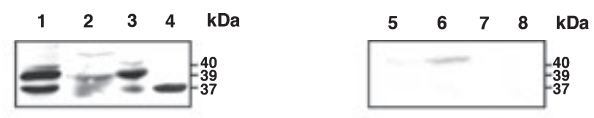

(b)

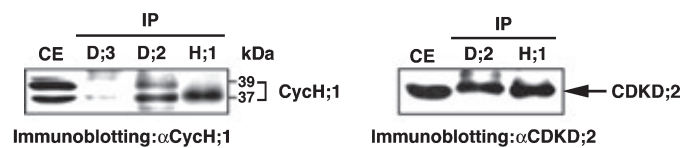

Immunoblotting: $\alpha \mathrm{CycH} ; 1$

Immunoblotting: $\alpha$ CDKD;2

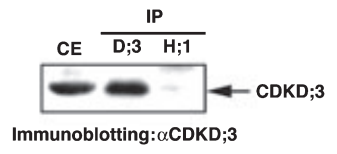

IP

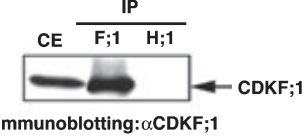

(c)

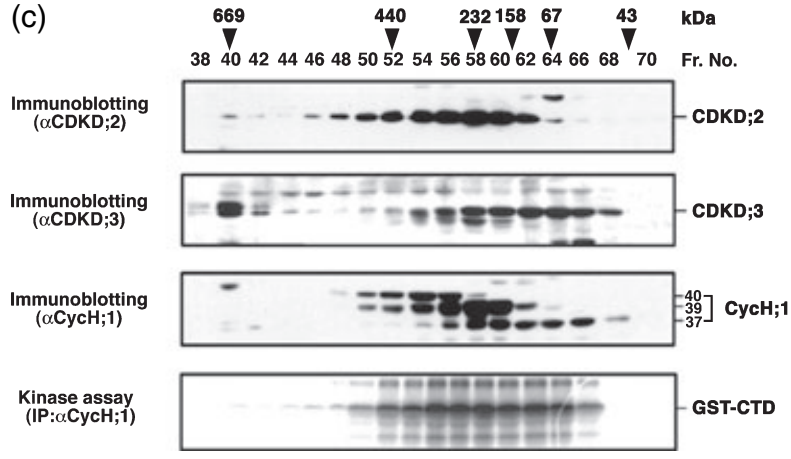

Figure 1. The $\mathrm{CycH} ; 1$ complexes in Arabidopsis.

(a) Immunoblotting of $\mathrm{CycH} ; 1$. Protein extracts $(30 \mu \mathrm{g})$ from Arabidopsis suspension cells (lanes 1 and 5), shoots (lanes 2 and 6) and roots (lanes 3 and 7) of seedlings, and from yeast cells overexpressing $\mathrm{CycH} ; 1$ (lanes 4 and 8) were immunoblotted with either the anti- $\mathrm{CycH} ; 1$ antibody (lanes 1-4) or with the antiserum depleted with nickel sepharose resin carrying $\mathrm{His}-\mathrm{CycH} ; 1$ (lanes 5-8).

(b) In vivo interaction between $\mathrm{CycH} ; 1$ and either $\mathrm{CDKD}$ or $\mathrm{CDKF} ; 1$. Protein extracts $(200 \mu \mathrm{g})$ from suspension cells were immunoprecipitated with antibodies recognizing $\mathrm{CDKD} ; 2(\mathrm{D} ; 2), \mathrm{CDKD} ; 3(\mathrm{D} ; 3), \mathrm{CDKF} ; 1(\mathrm{~F} ; 1)$ or $\mathrm{CycH} ; 1$ $(\mathrm{H} ; 1)$; and the immunoprecipitates (IP) were immunoblotted with the indicated antibody. As a control, $15 \mu \mathrm{g}$ of crude extract (CE) was loaded on an SDSPAGE gel.

(c) CAK complexes in suspension cells. Protein extracts $(40 \mathrm{mg})$ from suspension cells were fractionated using Sephacryl S300 gel exclusion chromatography, and $15 \mu \mathrm{l}$ of each fraction was immunoblotted with antibodies recognizing CDKD;2, CDKD;3 or $\mathrm{CycH} ; 1$. A sample of $150 \mu \mathrm{l}$ of each fraction was immnoprecipitated with the anti-CycH;1 antibody and assayed for kinase activity by using glutathione-S-transferase (GST)-CTD as the substrate. Arrowheads indicate the elution positions of marker proteins with their molecular masses.

detected in a range from 50 to $250 \mathrm{kDa}$ (fraction No. 56-66), which overlaps with the fractions including CDKD;2 and CDKD;3 (Figure 1c). In contrast, the $39-k D a$ protein was included in fraction no. 50-62 (120-480 kDa), which corresponded well to those of CDKD;2 (Figure 1c) suggesting that it may specifically bind CDKD;2. This is consistent with the result that the $39-\mathrm{kDa}$ protein was included in the immunoprecipitate with the anti-CDKD;2 antibody (Figure 1b). A kinase assay showed that the $\mathrm{CycH} ; 1$ complex in fraction No. 50-66 exhibited kinase activity towards the glutathione- $S$ transferase (GST) fusion of Arabidopsis RNA polymerase II CTD (Figure 1c), but not towards the GST-fused human CDK2 (data not shown); this indicates that both the 37- and
39-kDa proteins are associated with CTD kinase activity. The nature of the 40-kDa protein remains unknown; it formed a larger complex than either the 37- or 39-kDa proteins.

The kinase activity associated with $\mathrm{CycH} ; 1$ was further investigated in a heterologous system by using tobacco BY2 cells. $\mathrm{CycH} ; 1$ fused to green fluorescent protein (GFP) was expressed under the control of an estrogen-regulated promoter that responds to a derivative of estrogen, namely, $\beta$-estradiol (Zuo et al., 2000). Immunoprecipitation and immunoblotting with the anti-GFP antibody allowed detection of a 64-kDa protein that was also recognized by the anti$\mathrm{CycH} ; 1$ antibody (Figure 2a), suggesting that the anti-GFP antibody specifically precipitated $\mathrm{CycH} ; 1-$ GFP. The immunoprecipitates displayed high CDK2 and CTD kinase activities (Figure 2b), indicating that $\mathrm{CycH} ; 1-\mathrm{GFP}$ bound and activated tobacco CAK(s). In cell cultures treated with $1 \mu \mathrm{m}$ $\beta$-estradiol for $32 \mathrm{~h}$, the GFP fluorescence was detected in both the cytoplasm and the nucleus (Figure 2c). When transformed BY2 cells were synchronized with aphidicolin, the distribution of the GFP fluorescence did not vary throughout the cell cycle (data not shown). The transient expression of GFP-fused $\mathrm{CycH} ; 1$ in onion (Allium cepa) epidermal cells showed a similar localization in the cytoplasm and nuclei (Figure 2d).

\section{The WEE1 kinase downregulates CDKDs by tyrosine phosphorylation}

The amino acid sequences of all A- and B-type CDKs in Arabidopsis possess conserved Thr and tyrosine (Tyr) residues in the ATP-binding site (Figure 3a); these residues may be the targets for phosphorylation by the WEE1 kinase. However, we found that CDKDs also have the typical Thr/Tyr residues (Thr21 and Tyr22 on CDKD;1; Thr23 and Tyr24 on CDKD;2; and Thr22 and Tyr23 on CDKD;3), whereas they are not conserved in vertebrate CAKs (Figure 3a). This raised an interesting question - are plant CDKDs controlled by the WEE1 kinase?

As CDK phosphorylation by WEE1 has not been demonstrated in plants, we first tested whether Arabidopsis WEE 1 could phosphorylate CDKA; 1 in vitro. The GST-fused WEE1 efficiently phosphorylated GST-CDKA; 1 but not GST-CDKA; 1 carrying a substitution of Tyr15 with phenylalanine (Phe) (Figure 3b). No phosphorylation was observed with the control GST protein. This indicated that WEE1 could phosphorylate Tyr15 on monomeric CDKA;1. Similar assays were conducted using maltose binding protein (MBP)-fused CDKD;1, CDKD;2 and CDKD;3 as substrates. To exclude autophosphorylation, a lysine (Lys) residue in the catalytic cleft of each CDKD was changed to arginine (Arg). GSTWEE1 phosphorylated MBP-CDKD;2 and MBP-CDKD;3 but neither MBP-CDKD;1 nor the control MBP protein (Figure $3 b, c)$. The phosphorylation signal disappeared when either Tyr24 of CDKD;2 or Tyr23 of CDKD;3 was substituted 
(a)

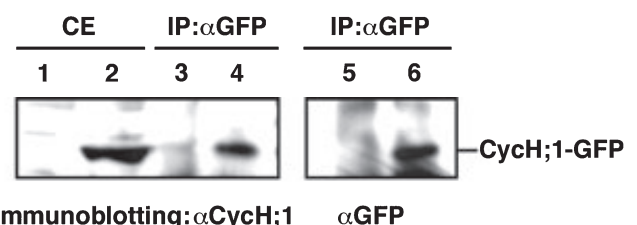

(b)

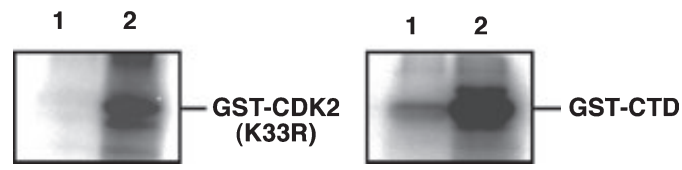

(c)
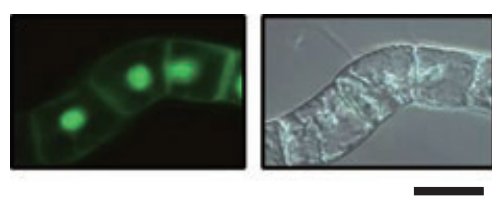

(d)

GFP-CycH;1
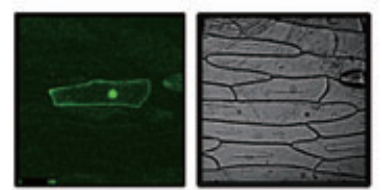

CycH;1-GFP
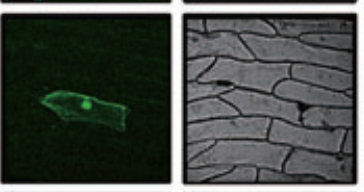

WEE1-GFP
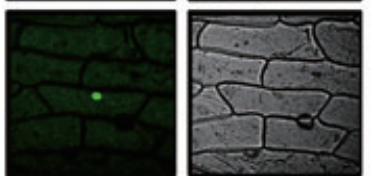

Figure 2. Expression of $\mathrm{CycH} ; 1-\mathrm{GFP}$ in tobacco $\mathrm{BY} 2$ and onion epidermal cells.

(a) Immunological detection of $\mathrm{CycH} ; 1-\mathrm{GFP}$ in tobacco BY2 cells. Transgenic BY2 cells were synchronized with aphidicolin and then treated with $1 \mu \mathrm{m} \beta$ estradiol for $32 \mathrm{~h}$ after release from the aphidicolin block. Protein extracts $(15 \mu \mathrm{g})$ from cells expressing either green fluorescent protein (GFP) alone (lanes 1, 3 and 5) or $\mathrm{CycH}_{1}$;-GFP (lanes 2, 4 and 6) were immunoblotted with the anti-CycH; 1 antibody (lanes 1 and 2), and $200 \mu \mathrm{g}$ of protein extracts was immunoprecipitated with the anti-GFP antibody and detected with either the anti-CycH;1 (lanes 3 and 4) or the anti-GFP (lanes 5 and 6) antibody, as indicated.

(b) CDK and CTD kinase activities of $\mathrm{CycH}$;1-GFP. Protein extracts $(200 \mu \mathrm{g})$ from cells either expressing GFP alone (lane 1) or $\mathrm{CycH} ; 1-\mathrm{GFP}$ (lane 2) were immunoprecipitated with the anti-GFP antibody, and the immunoprecipitates were subjected to a kinase assay by using either GST-CDK2 (K33R) or GST-CTD as the substrate. To exclude the possibility of autophosphorylation, the lysine residue in the catalytic domain of CDK2 was substituted with arginine (K33R). (c) Subcellular localization of $\mathrm{CycH} ; 1-\mathrm{GFP}$ in tobacco BY2 cells. Fluorescent microscopic images of $\mathrm{CycH}$;1-GFP (left) and the corresponding differential interference contrast (DIC) images (right) are shown. Scale bar $=40 \mu \mathrm{m}$. (d) Subcellular localization of GFP-fused $\mathrm{CycH}$;1 and WEE1 in onion epidermal cells. Fluorescent (left) and bright-field (right) images of cells are shown. $\mathrm{CycH} ; 1$ fused to GFP at the amino terminus and the carboxy terminus were tested. Scale bar $=100 \mu \mathrm{m}$.

with Phe. These results suggested that the conserved Tyr residues of CDKD;2 and CDKD;3 are targeted by WEE1. CDKF; 1 has Tyr32 in the corresponding region; however, the
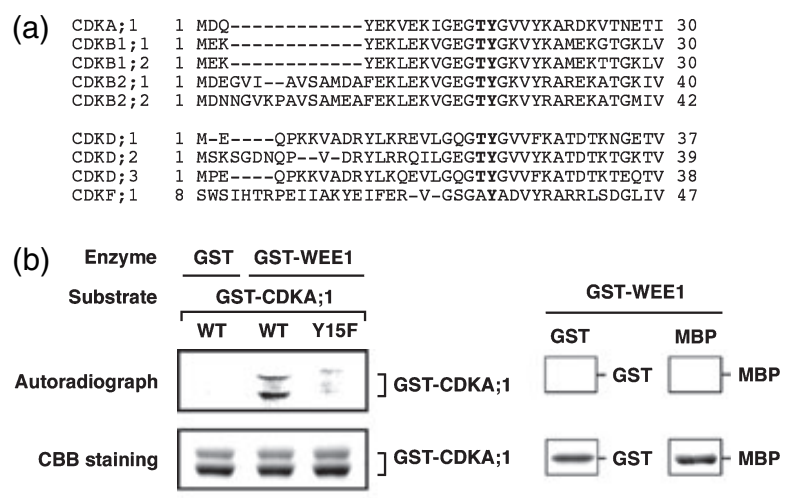

(c) Enzyme GST $\begin{gathered}\text { GST- } \\ \text { WEE1 }\end{gathered}$ GST GST- WEE1 GST GST-
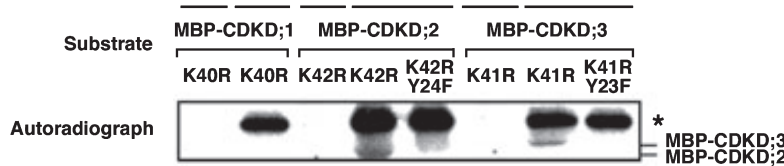

CBB staining

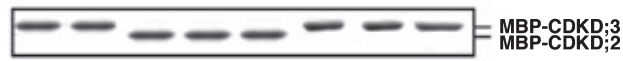

(d)

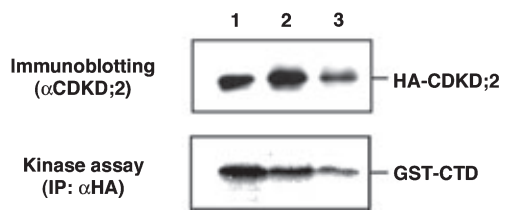

Figure 3. Arabidopsis WEE1 phosphorylates CDKDs as well as CDKA;1.

(a) Alignment of amino-terminal amino acids of Arabidopsis CDKs and CAKs. The conserved threonine $(\mathrm{T})$ and tyrosine $(\mathrm{Y})$ residues in the ATP-binding site are indicated by bold letters. Numbers indicate amino acid positions. Dashes represent gaps introduced to give maximal identity.

(b) Phosphorylation of CDKA;1 by WEE1. GST-CDKA;1 [either wild-type (WT) or Y15F mutant] was incubated with $10 \mathrm{ng}$ of either glutathione-S-transferase (GST) or GST-WEE1 in the presence of $\left[\gamma_{-}{ }^{32} \mathrm{P}\right]$ ATP. Either GST or maltose binding protein (MBP) alone were used as controls.

(c) Phosphorylation of CDKD;2 and CDKD;3 by WEE1. MBP-CDKDs were reacted with $10 \mathrm{ng}$ of either GST or GST-WEE1. To exclude autophosphorylation of the substrates, each lysine $(K)$ residue in the catalytic domain of CDKDs was substituted with arginine (R). To determine the phosphorylation sites, $\mathrm{Y} 24$ in CDKD;2 and $\mathrm{Y} 23$ in CDKD;3 were substituted with phenylalanine (F). An asterisk indicates the autophosphorylation signal of GST-WEE1.

(d) WEE1 downregulates CDKD;2 in Arabidopsis root protoplasts. CDKD;2 was co-expressed with either an empty vector pMESHI (lane 1) or with $10 \mu \mathrm{g}$ (lane 2) or $50 \mu \mathrm{g}$ (lane 3 ) of pMESHI-WEE1. Total protein $(10 \mu \mathrm{g})$ was immunoblotted with the anti-CDKD;2 antibody. Protein extract $(100 \mu \mathrm{g})$ was immunoprecipitated with the anti-hemagglutinin (anti-HA) antibody, followed by a phosphorylation reaction using GST-CTD as a substrate.

preceding Thr is missing (Figure 3a). However, as CDKF;1 showed high autophosphorylation activity, we could not examine Tyr32 phosphorylation.

To test whether WEE1 inhibits CDKD activity in plant cells, we co-expressed c-myc and hemagglutinin (HA) epitopetagged forms of WEE1 and CDKD, respectively, in Arabidopsis root protoplasts. As CDKD;3 was not expressed in the protoplasts for unknown reasons, only CDKD;2 was assessed for CTD kinase activity by using immunoprecipitates 
with an anti-HA antibody. The enzyme activity was decreased by co-expression with myc-WEE1 (Figure 3d, lane 2 ), and this result was reproducible, suggesting that WEE1 downregulated CDKD;2 in the protoplasts. Increasing the expression level of myc-WEE1 resulted in the lower accumulation of HA-CDKD;2 (Figure 3d, lane 3); thus, we failed to compare the kinase activities. GFP-fused WEE1 displayed nearly exclusive nuclear localization in $A$. cepa epidermal cells (Figure 2d).

\section{CDKF; 1 is involved in CDK activation in plant cells}

We identified Arabidopsis mutants of $C D K D ; 1$ and $C D K D ; 3$ from T-DNA insertion collections. T-DNAs were inserted into the second exon of $C D K D ; 1$ and the third exon of $C D K D ; 3$, as shown in Figure 4a. Each mutant was backcrossed with wild-type plants three times, and a homozygous line containing a single T-DNA insertion was established by genomic Southern hybridization with a labeled T-DNA probe (data not shown). RT-PCR with the CDKD;1 mutant (hereafter called $c d k d ; 1-1)$ showed that the cDNA upstream to the TDNA insertion site was amplified from the mRNA of shoots and roots; however, the downstream region was not amplified at all (Figure 4b). RT-PCR with the CDKD;3 mutant (hereafter called $c d k d ; 3-1$ ) showed that neither the cDNA upstream nor downstream to the T-DNA insertion site was amplified (Figure 4b). Both mutants showed no developmental defects throughout the life cycle under normal growth conditions (Figure 4c), suggesting that CDKD;1 and $\mathrm{CDKD} ; 3$ do not play an essential role in plant development.

In Arabidopsis, CDKD;3 and CDKF;1 were assumed to be the major kinases that catalyze the T-loop phosphorylation of CDKs (Shimotohno et al., 2003, 2004; Umeda et al., 1998). However, as described above, CDKD;3 was not essential for plant growth, leading to the proposal that CDKF; 1 is responsible for the majority of the CDK kinase activities in Arabidopsis cells. Previously, we reported that CDKF;1 phosphorylates and activates human CDK2 in vitro (Umeda et al., 1998); however, in vivo interaction with endogenous Arabidopsis CDKs remains unknown. Here, we co-expressed myc-CDKF; 1 and HA-CDKA;1 in Arabidopsis root protoplasts, and the immunoprecipitates with the anti-HA antibody were subjected to a kinase assay. As shown in Figure 5a, HA-CDKA;1 displayed histone H1-kinase activity only when myc-CDKF; 1 was co-expressed, indicating that CDKA; 1 required $C D K F ; 1$ to exert its enzyme activity.

CDKF; 1 also has a conserved Thr residue (Thr290) within the T-loop (Umeda et al., 1998). This prompted us to examine whether T-loop phosphorylation is required for CDKF; 1 activity. When myc-CDKF; 1 carrying the substitution of Thr290 with alanine was co-expressed with HA-CDKA; 1 in root protoplasts, HA-CDKA;1 did not exhibit kinase activity at all (Figure 5a). We then expressed either the wild-type or the T290A mutant of myc-CDKF;1 in root protoplasts, and its (a)
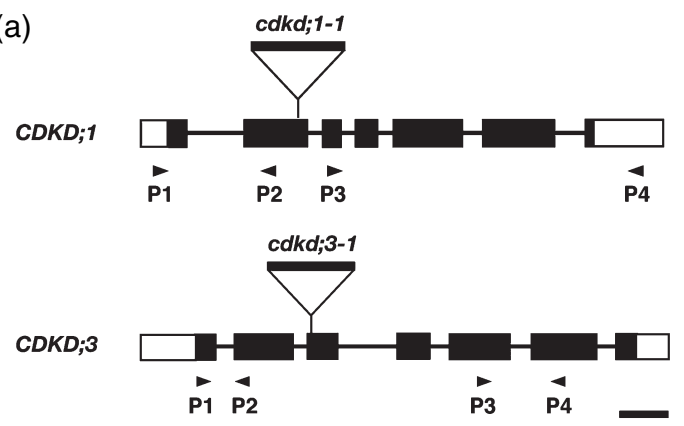

(b)
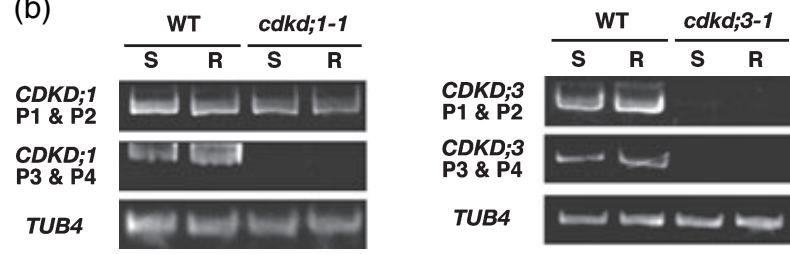

(c)
WT

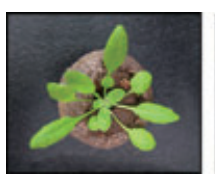

cdkd;1-1

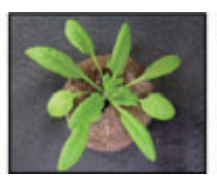

cdkd;3-1

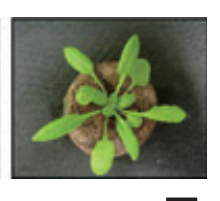

Figure 4. T-DNA insertion mutants of $C D K D ; 1$ and $C D K D ; 3$.

(a) Schematic diagrams of the $C D K D ; 1$ and $C D K D ; 3$ genes. Exons and introns are indicated by boxes and solid bars, respectively. Black and open boxes represent coding and non-coding regions, respectively, on which the T-DNA insertion sites are shown. Arrowheads indicate primers that were used for RTPCR. Scale bar $=250 \mathrm{bp}$.

(b) Expression analysis of the mutants. RT-PCR was conducted with total RNA from shoots (S) and roots (R) of either wild-type (WT) or mutant seedlings by using the indicated sets of primers. The amplified cDNAs were stained with ethidium bromide. TUBULIN 4 (TUB4) was used as a control.

(c) Eighteen-day-old seedlings of WT and T-DNA insertion mutants. Scale bar $=1 \mathrm{~cm}$.

activity was tested by using GST-CDK2 as a substrate. The result showed that the T-loop mutation significantly reduced the kinase activity of myc-CDKF; 1 (Figure $5 \mathrm{~b}$ ). These results suggest that the phosphorylation of Thr290 on CDKF; 1 is indispensable for CDK activation in plant cells.

\section{Discussion}

Our results showed that $\mathrm{CycH} ; 1$ forms a stable complex with CDKD;2 in Arabidopsis cells. GFP-fused $\mathrm{CycH} ; 1$ was localized in the cytoplasm and nuclei, similar to the localization of CDKD;2 (Shimotohno et al., 2004). This is in contrast to other organisms where cyclin $\mathrm{H}$ and CAK complexes are exclusively localized to the nucleus (Jordan et al., 1997; Kaldis, 1999; Krempler et al., 2005). During seed germination in Arabidopsis, transcripts of $\mathrm{CycH} ; 1$ and $C D K D ; 2$ accumulate before the activation of cell division in the root apex (Menges et al., 2005). The first signs of germination are the 
(a)

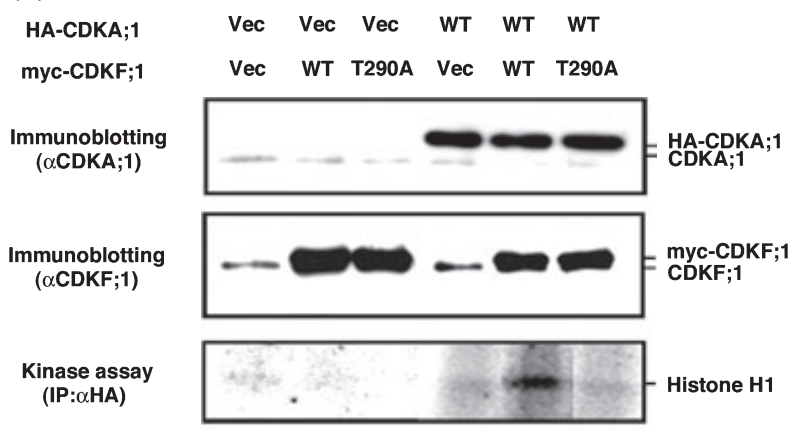

(b)

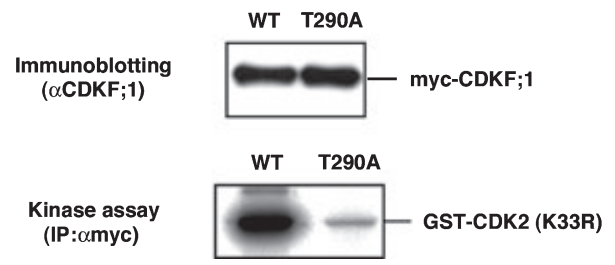

Figure 5. T-loop phosphorylation of CDKF; 1 is indispensable for CAK activity.

(a) Histone $\mathrm{H} 1$ kinase activity of CDKA;1 in Arabidopsis root protoplasts. HACDKA;1 and/or myc-CDKF; [ [wild-type (WT) or T290A mutant] were expressed in root protoplasts, and $10 \mu \mathrm{g}$ of crude extracts was immuoblotted with either the anti-CDKA; 1 or the anti-CDKF;1 antibody. As a control, pMENCHU and/or pMESH vectors $(\mathrm{Vec})$ were introduced into root protoplasts. Endogenous CDKA;1 and CDKF; 1 were also detected. Protein extracts $(100 \mu \mathrm{g})$ were immunoprecipitated with the anti-hemagglutinin (anti-HA) antibody and assayed for histone $\mathrm{H} 1$ kinase activity.

(b) Loss of CDKF; 1 activity by T-loop mutation. Either WT or the T290A mutant of myc-CDKF; 1 was expressed in Arabidopsis root protoplasts, and $5 \mu \mathrm{g}$ of crude extracts was immunoblotted with the anti-CDKF; 1 antibody. Crude extracts $(100 \mu \mathrm{g})$ were immunoprecipitated with the anti-c-myc antibody, and the immunoprecipitates (IP) were subjected to a kinase assay in which glutathione-S-transferase (GST)-CDK2 (K33R) was used as the substrate.

resumption of essential transcription, DNA repair or other metabolic processes. Therefore, the $\mathrm{CycH} ; 1-\mathrm{CDKD} ; 2 \mathrm{com}$ plex may be involved in transcription via CTD phosphorylation during early seed germination. We failed to observe a tight interaction between $\mathrm{CycH} ; 1$ and $\mathrm{CDKD} ; 3$. This was surprising because $\mathrm{CycH} ; 1$ interacted with $\mathrm{CDKD} ; 3$ in a yeast two-hybrid assay, and it enhanced the kinase activity in insect cells (Shimotohno et al., 2004). The 37-kDa protein of $\mathrm{CycH} ; 1$ formed a protein complex of $\sim 100 \mathrm{kDa}$ in gel filtration fractions (fraction no. 62-66) that contained a significant quantity of CDKD;3 (Figure 1c). Moreover, the GFP-fused $\mathrm{CycH} ; 1$, which was overexpressed in tobacco BY2 cells and immunoprecipitated with the anti-GFP antibody, displayed not only CTD but also CDK kinase activity, an indication that the CAK activity was efficiently recovered with the GFP tag. It is probable that the $\mathrm{CycH} ; 1-\mathrm{CDKD} ; 3$ complex is not as stable as the $\mathrm{CycH} ; 1-\mathrm{CDKD} ; 2$ complex in vivo; thus, it might be dissociated during immnoprecipitation with the anti-CycH;1 antibody, which might recognize the $\mathrm{CycH} ; 1-\mathrm{CDKD} ; 3$ complex less efficiently.
The anti- $\mathrm{CycH} ; 1$ antibody recognized three Arabidopsis proteins of 37,39 and $40 \mathrm{kDa}$. The $37-\mathrm{kDa}$ protein was considered as the intrinsic $\mathrm{CycH} ; 1$ because the recombinant protein that was expressed in yeast cells showed the same mobility on SDS-PAGE. Immunoprecipitation experiments showed that both the $37-$ and $39-\mathrm{kDa}$ proteins bound CDKD;2. However, gel filtration chromatography resolved the $37-\mathrm{kDa}$ protein in fractions that overlapped with those of CDKD;2 and CDKD;3, whereas the peak fraction (fraction No. 58-60) of the 39-kDa protein was almost the same as that of CDKD;2. This suggests that the $39-\mathrm{kDa}$ protein may have a higher affinity to CDKD;2. In mammals, two kinases - cyclin C-CDK8 and CK2 - have been identified that phosphorylate cyclin $\mathrm{H}$ and regulate its activity (Akoulitchev et al., 2000; Schneider et al., 2002). However, a protein phosphatase treatment caused no mobility shift of $\mathrm{CycH} ; 1$ on SDS-PAGE (data not shown), suggesting that a protein modification other than phosphorylation may produce the $\mathrm{CycH} ; 1$ variants (Krempler et al., 2005). Note that it is still possible that the 39- and 40-kDa bands are not associated with $\mathrm{CycH} ; 1$ isoforms, but rather with other proteins that contain epitope(s) similar to that of $\mathrm{CycH} ; 1$.

The protein kinase WEE1 was described in fission yeast, where mutated cells showed a small cell (wee) phenotype caused by premature entry into mitosis (Featherstone and Russell, 1991; Russell and Nurse, 1987). WEE1 is encoded at a single locus in the Arabidopsis genome, and its overexpression in fission yeast caused cells to elongate without cell division (Sorrell et al., 2002). A similar result was also reported with a maize WEE1 homologue that was able to inhibit the activity of $\mathrm{p} 13^{\text {suc1 }}$-adsorbed CDK from maize (Sun et al., 1999). However, the direct link between CDKs and WEE1 has not been demonstrated in plants. Here, we reported that Arabidopsis WEE1 phosphorylated a specific Tyr residue within the ATP-binding site of CDKA; 1 in vitro, suggesting that it has the same specificity as yeast and vertebrate WEE1 (Featherstone and Russell, 1991; Gould and Nurse, 1989; Lundgren et al., 1991; Parker and PiwnicaWorms, 1992; Parker et al., 1992). As recombinant CDKA;1 was expressed in Escherichia coli, Arabidopsis WEE1 may have the ability to phosphorylate monomers of CDKA;1. To our knowledge, there is no report of WEE 1 phosphorylating CDK monomers in fission yeast and vertebrates (Parker et al., 1992).

Interestingly, WEE1 also phosphorylated CDKD;2 and CDKD;3 in vitro, and CDKD;2 kinase activity was inhibited by WEE1 in Arabidopsis root protoplasts. This indicates that CAK activity is regulated by WEE1. Arabidopsis WEE 1 is strongly S-phase regulated (Menges et al., 2005); thus, it is likely that CDKD activities may be downregulated in the $S$ phase. Our mutational analyses revealed that WEE1 targeted the specific Tyr residues of CDKD;2 and CDKD;3. The Thr and Tyr residues within the ATP-binding site are conserved not only in Arabidopsis CDKDs but also in other plant CDKDs. 
Therefore, CDKD phosphorylation by WEE1 may be a common regulatory mechanism of plant CAKs. Although $\mathrm{CDK} 7 / \mathrm{p} 40^{\mathrm{MO} 15}$-related proteins in vertebrates lack the Thr/ Tyr motif, those in budding and fission yeasts possess conserved residues, namely, Thr17 and Tyr18 on Kin28p and Thr21 and Tyr22 on Mcs6. This suggests that they may be also controlled by WEE1 kinases. CDKD;1 also has the typical Thr/Tyr motif but was not phosphorylated by WEE 1 in our assay. This distinct feature of CDKD;1 was noted in the T-loop phosphorylation; CDKF;1 phosphorylated CDKD;2 and CDKD;3 but not CDKD;1 regardless of the conserved Thr residue within the T-loop (Shimotohno et al., 2004).

We observed previously that $C D K D ; 3$ and $C D K F ; 1$, but neither $C D K D ; 1$ nor $C D K D ; 2$, were able to suppress the cak $7^{\text {ts }}$ mutation of budding yeast (Shimotohno et al., 2003). In fact, a significant level of CDK kinase activity was detected with CDKD;3 and CDKF;1, but not with CDKD;1 and CDKD;2 (Shimotohno et al., 2003, 2004; Umeda et al., 1998). Here, we demonstrated that a knockout mutant of $C D K D ; 3$ showed no defect in plant development. In contrast, a homozygous mutant of CDKF; 1 showed a severe defect under the same growth conditions (C.K. and M.U., unpublished data). Therefore, it is likely that $\mathrm{CDKF} ; 1$ plays a major role in CDK phosphorylation and activation. The CDK kinase activity of recombinant CDKF;1 produced in insect cells was 10 -fold higher than that of CDKD;3 (Shimotohno et al., 2004), thus supporting the above idea. Based on these results, we propose a model for CDK and CTD phosphorylation in Arabidopsis: the Tloop of CDKs is phosphorylated by CDKF;1, and CTD is phosphorylated by $\mathrm{CyCH} ; 1-\mathrm{CDKD} ; 2$, which is negatively regulated by the WEE1 kinase (Figure 6). $\mathrm{CyCH} ; 1-\mathrm{CDKD} ; 2$ also exhibits CDK kinase activity, but its level is significantly lower than that of CDKF;1 (Shimotohno et al., 2003, 2004). The manner in which different kinases are engaged in CDK and CTD phosphorylation is similar to that observed in budding yeast; Kin28p phosphorylates the CTD (Cismowski et al., 1995; Feaver et al., 1994), and Cak1p phosphorylates and activates Cdc28p (Espinoza et al., 1996; Kaldis et al., 1996; Thuret et al., 1996). Kimmelman et al. (1999) reported that Cak1p is also involved in basal transcription through Kin28p phophorylation. Similarly, Arabidopsis CDKF; 1 phosphorylates Ser162 and Thr168 within the T-loop of CDKD;2 and activates its CTD kinase activity (Figure 6; Shimotohno et al., 2004; Umeda et al., 2005). Therefore, despite the low sequence similarity, $\mathrm{CDKF} ; 1$ is functionally related to budding yeast Cak $1 \mathrm{p}$. This is supported by recent biochemical studies on CAKs (Tsakraklides and Solomon, 2002); Cak1p and CDKF;1 displayed a preference for cyclin-free CDK substrates, were insensitive to the protein kinase inhibitor 5 '-fluorosulfonylbenzoyladenosine (FSBA), and were insensitive to the mutation of a highly conserved Lys residue found in

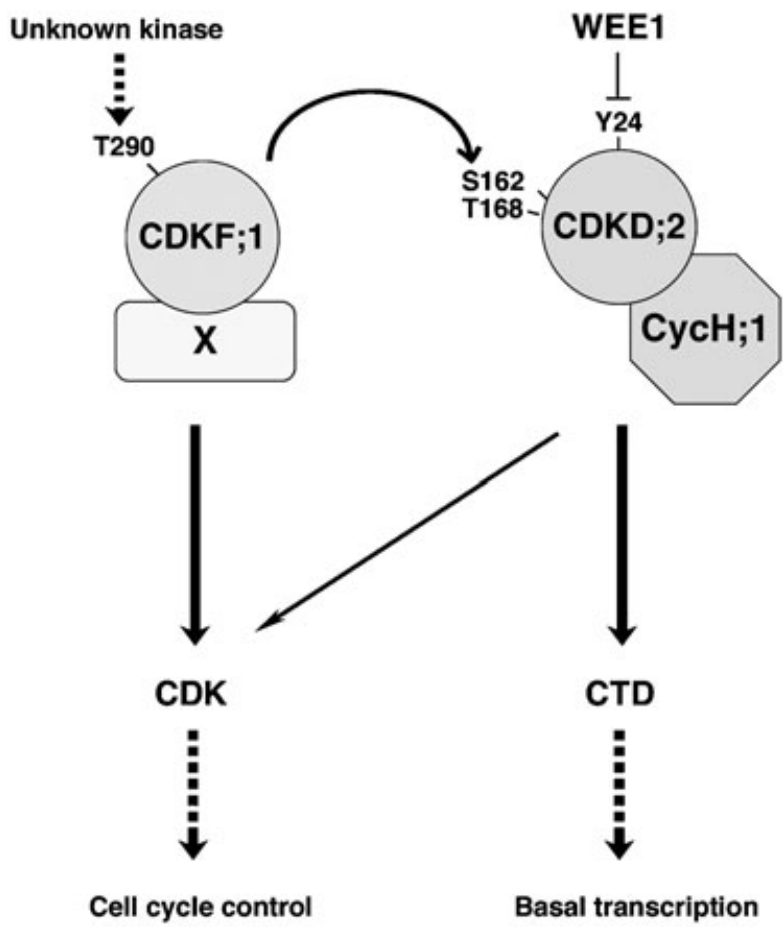

Figure 6. Phosphorylation of CDK and CTD in Arabidopsis.

CDKF; 1 is activated by phosphorylation of T290 within the T-loop and exerts CDK kinase activity to control the cell cycle. CDKF; 1 forms an active $130-k D a$ complex in Arabidopsis; hence, some unknown subunit(s) (X) may be included in the complex. Another activity of CDKF;1 is to phosphorylate and activate CDKD;2, which forms a stable complex with $\mathrm{CycH} ; 1$ to display CTD kinase activity. The CDK kinase activity of $\mathrm{CycH} ; 1-\mathrm{CDKD} ; 2$ is significantly lower than that of CDKF;1. CDKD;2 is downregulated by WEE1, which phosphorylates $\mathrm{Y} 24$ in the ATP-binding site of CDKD;2.

the nucleotide binding pocket. Moreover, Kaldis et al. (1998) revealed that Cak1p was localized in both the nucleus and cytoplasm in a manner similar to CDKF; 1 . Conservation of these unusual properties in budding yeast and Arabidopsis may indicate shared evolutionary requirements in cell-cycle regulation and transcription.

In Arabidopsis root protoplasts, CDKA; 1 was active when it was co-expressed with CDKF;1. This clearly shows that a plant CDK requires CAK for its activity. Using database searches, CDKF; 1 homologues were found only in plant species, namely Euphorbia, rice (Oryza sativa) and soybean (Glycine max), but not in other kingdoms (Umeda et al., 2005). All the four plant CDKFs contain the phosphoregulatory site within the T-loop. A mutation of Thr290 in the T-loop region of CDKF; 1 dramatically reduced CAK activity, suggesting that T-loop phosphorylation is essential for CDKF activity in vivo (Figure 6). Therefore, the identification of an upstream kinase will be particularly interesting because it will link internal and/or external signals to cellcycle machinery that governs not only cell proliferation but also cell differentiation during plant development. 


\section{Experimental procedures}

\section{Plant material}

Arabidopsis thaliana (ecotype Columbia) plants were grown at $23^{\circ} \mathrm{C}$ under continuous light conditions. For the isolation of protoplasts, 50-70 root segments from 10-day-old seedlings grown on Murashige and Skoog (Sigma, St Louis, MO, USA) agar plates were used. An Arabidopsis cell suspension culture was maintained as described previously (Glab et al., 1994). Tobacco BY2 cells were maintained in a modified Murashige and Skoog medium as described by Nagata et al. (1992). Total protein was extracted from the suspension cells 4 days after subculture (Magyar et al., 1997).

\section{Identification of T-DNA insertion mutants}

The T-DNA insertion mutants of $C D K D ; 1$ and $C D K D ; 3$ were isolated from the collections of the Max-Planck-Institute für Züchtungsforschung (Ríos et al., 2002) and The Salk Institute, respectively. Seed stock numbers of cdkd;1-1 and cdkd;3-1 are MPI8258 and SALK_120536, respectively. The insertions were examined by genomic PCR with Ex Taq DNA polymerase (TaKaRa, Tokyo, Japan) by using a set of primers that hybridize to the T-DNA and each CDKD; $5^{\prime}$ CTGGGAATGGCGAAATCAAGGCATC- $3^{\prime}$ and $5^{\prime}$-GTTGCTGATAGGTATCTAAAGCGAGAGGT-3' were used for $c d k d ; 1-1$ and $5^{\prime}$-GGATTTTCGCCTGCTGGGGCAAACCAGCGT- $3^{\prime}$ and $5^{\prime}$-CAGCCAAAGAAAGTTGCTGATAGGTATCTC-3' for $c d k d ; 3-1$. The nucleotide sequences of the amplified fragments were determined to identify the T-DNA insertion site. Each line was backcrossed with wild-type plants three times. The Titanium One-Step RT-PCR kit (BD Biosciences Clontech, San Diego, CA, USA) was used for RT-PCR with total RNA from shoots and roots of 21-day-old seedlings. In a total reaction volume of $10 \mu \mathrm{l}, 0.1 \mu \mathrm{g}$ of RNA and the following primers were used: either $5^{\prime}$-TTGCTTGTGATCCGATTAGAGAGTT-3' (P1) and 5'-CATCACCAGGCGAAAGATAGAGATT-3' (P2) or 5'-CCAAACAACTTGTTGATAGGACCCA-3' (P3) and 5'-TAGACAGAAAGAATGATTCAAACTA-3' (P4) for $c d k d ; 1-1$; and either 5'-CGAATTCGTCGACATGCCGGAGCAGCC-3' (P1) and 5'-GAACTCAAAGACAAGATGCA$3^{\prime}$ (P2) or 5'-AGTGGCCGGATTTAACAAAG-3' (P3) and 5'-TCCTTGTCAACATGAGACTT-3' (P4) for $c d k d ; 3-1$. As a control, TUB4 cDNA was amplified by using primers $5^{\prime}$-CTCTGTGCATCAGCTTGTCGAAAACG- $3^{\prime}$ and 5'-CCGAGGGAGCCATTGACAACATCTT- $3^{\prime}$. The PCR conditions were one cycle at $50^{\circ} \mathrm{C}$ for $60 \mathrm{~min}$ and at $94^{\circ} \mathrm{C}$ for $5 \mathrm{~min} ; 35$ cycles at $94^{\circ} \mathrm{C}$ for $30 \mathrm{sec}$, at $65^{\circ} \mathrm{C}$ for $30 \mathrm{sec}$ and at $68^{\circ} \mathrm{C}$ for $1 \mathrm{~min}$; and one cycle at $68^{\circ} \mathrm{C}$ for $2 \mathrm{~min}$.

\section{Plasmid construction}

The coding regions of $W E E 1$ and $\mathrm{CyCH} ; 1$ were amplified by PCR and cloned into the GATEWAY entry vector pENTR/D-TOPO (Invitrogen, San Diego, CA, USA). Recombination reactions were performed between the entry clones and the destination vectors pDEST15 and pDEST17 by using LR Clonase (Invitrogen) to produce His-tagged $\mathrm{CycH} ; 1$ and GST-fused WEE1, respectively. The destination vector pYES-DEST52 (Invitrogen) was used to express $\mathrm{CycH} ; 1$ in $\mathrm{Sac}$ charomyces cerevisiae strain YPH500. Yeast protein extracts were prepared as described previously (Vitaly, 2000). For transient expression in $A$. cepa epidermal cells, the destination vectors either pGWB5 or pGWB6 (gifts from Dr Nakagawa, Shimane University, Matsue, Japan) were used to produce WEE1-GFP, $\mathrm{CycH}$;1-GFP (or GFP-CycH;1), respectively. To express $\mathrm{CycH} ; 1-\mathrm{GFP}$ in an estrogeninducible manner, the $\mathrm{CyCH} ; 1$-GFP fragment with the Xhol site at the $\mathrm{N}$-terminal end and the Spel site at the C-terminal end was PCR- amplified and cloned into the Xhol/Spel sites of pER8 (Zuo et al., 2000). For expression in Arabidopsis root protoplasts, cDNAs of WEE1, CDKF;1 and CDKA;1 were cloned into either the Sall site of pMESHI or the EcoRI site of pMENCHU (Ferrando et al., 2000, 2001) to produce myc-WEE1, myc-CDKF;1 and HA-CDKA;1, respectively. Nucleotide substitutions were introduced using a Mutan-Super Express $\mathrm{Km}$ kit (TaKaRa). cDNAs of CDKA;1,CDKD;2 and CDKD;3 were cloned into the EcoRI site of pGEX-5X-1 (Amersham Biosciences, Piscataway, NJ, USA) and either the BamHI/Sall or the EcoRl site of pMAL-c2X (New England BioLabs, Beverly, MA, USA) to produce GST-CDKA;1, MBP-CDKD;2 and MBP-CDKD;3, respectively. The other constructs have been described previously (Shimotohno et al., 2004).

\section{Protein expressions in Escherichia coli}

pGEX and pDEST15 vectors were transformed into the $E$. coli strain of either BL21 or BL21-Al (Invitrogen), respectively. The transformants were grown at $27^{\circ} \mathrm{C}$ to an $\mathrm{OD}_{600}$ of $0.6-0.8$, followed by induction with either $0.2 \mathrm{~mm}$ isopropyl $\beta$-D-thiogalactoside (IPTG) or $0.2 \%$ L-arabinose, and allowed to continue culturing for 4-6 h. GST-fusion proteins were purified with glutathione-Sepharose $4 B$ (Amersham Biosciences). The purification of GST-fused human CDK2 (carrying the K33R mutation) and Arabidopsis CTD has been described previously (Poon et al., 1993; Umeda et al., 1998). MBP-fused proteins were expressed in E. coli BL21 cells; these cells were grown to an $\mathrm{OD}_{600}$ of $0.4-0.6$, followed by induction with $0.4 \mathrm{~mm}$ IPTG, allowed to continue culturing for a further $5 \mathrm{~h}$, and were then purified with amylose resin (New England Biolabs). His-tagged $\mathrm{CycH} ; 1$ was expressed in E. coli BL21-Al cells, as described above, and purified using a nickel-Sepharose column (Qiagen, Hilden, Germany).

\section{Protein expressions in plant cells}

Agrobacterium-mediated transformation of tobacco BY2 cells was performed as described by lto et al. (1998). For synchronization, a 7-day-old culture was diluted 1:9, mixed with $5 \mathrm{mg} \mathrm{I}^{-1}$ aphidicolin, and cultured for $24 \mathrm{~h}$. Aphidicolin was then removed by washing the cells with the fresh medium to restart the cell cycle. In order to express $\mathrm{CycH} ; 1-\mathrm{GFP}, 1 \mu \mathrm{m} \beta$-estradiol was added to the culture just after release from the aphidicolin block. The preparation and transfection of Arabidopsis root protoplasts has been described previously (Abel and Theologis, 1994). Protoplasts $\left(2 \times 10^{5}\right.$ cells $)$ were transfected with $50 \mu \mathrm{g}$ of plasmid DNA and incubated at $22^{\circ} \mathrm{C}$ for $12 \mathrm{~h}$ under continuous illumination. Transient expression in A. cepa epidermal cells was conducted as described by Shimotohno et al. (2004). The GFP fluorescence was observed with a confocal laser scanning microscope system (MicroRadiance MR/AG-2; Bio-Rad, Hercules, CA, USA).

\section{Immunoblotting and kinase assay}

A polyclonal antibody was raised in rabbits against the His-tagged $\mathrm{CycH} ; 1$ produced in E. coli. The antiserum was purified with a HiTrap rProtein A FF affinity column (Amersham Biosciences) and then with a HiTrap $\mathrm{N}$-hydroxysuccinimide (NHS)-activated HP affinity column (Amersham Biosciences) that covalently bound His$\mathrm{CycH} ; 1$. Depletion of the antibody was performed with $\mathrm{His}-\mathrm{CycH} ; 1$ as described by Shimotohno et al. (2003). Immunoblotting was conducted by using an ECL Western Blotting Detection kit (Amersham Biosciences). Phosphatase treatment was performed with $200 \mathrm{U}$ of lambda protein phosphatase (New England Biolabs) in a buffer ( $50 \mathrm{~mm}$ Tris- $\mathrm{HCl}, 0.1 \mathrm{~mm} \mathrm{Na}{ }_{2}$ EDTA, $5 \mathrm{~mm}$ DTT, $0.01 \%$ polyoxy- 
ethylene lauryl ether, $\mathrm{pH} 7.5$ ) at $30^{\circ} \mathrm{C}$ for $1 \mathrm{~h}$. The kinase assay was performed as described previously (Shimotohno et al., 2004; Umeda et al., 1998). Immunoprecipitation was conducted either with specific antibodies or with the anti-HA (12CA) monoclonal antibody (Roche, Indianapolis, IN, USA), an anti-c-myc monoclonal antibody (Berkeley Antibody Company, Richmond, CA, USA) or an anti-GFP polyclonal antibody (Medical \& Biological Laboratories, Nagoya, Japan) as described by Umeda et al. (1998). Fractionation of Arabidopsis protein extracts by Sephacryl S300 gel exclusion chromatography was performed as described previously (Shimotohno et al., 2004). Elution profiles of proteins in LMW and HMW gel filtration calibration kits (Amersham Biosciences) were used for estimations of molecular mass.

\section{Acknowledgements}

We thank Dr Tsuyoshi Nakagawa (Shimane University) for the pGWB vectors and Dr Nam-Hai Chua (The Rockefeller University) for the pER8 vector. We are obliged to Dr Nobuhiro Tsutsumi and Dr Shin-ichi Arimura (The University of Tokyo) for their help with particle bombardment. We are grateful to the Arabidopsis Biological Resource Center at The Ohio State University for providing the seeds of the T-DNA insertion mutants (SALK_120536). This work was supported by a Grant-in-Aid for Scientific Research on Priority Areas (grant no. 15031210) and a Grant-in-Aid for Scientific Research (B) (grant no. 16370019). A.S. was supported by the Alexander von Humboldt Foundation Postdoctoral Fellowship.

\section{References}

Abel, S. and Theologis, A. (1994) Transient transformation of Arabidopsis leaf protoplasts: a versatile experimental system to study gene expression. Plant J. 5, 421-427.

Akoulitchev, S., Chuikov, S. and Reinberg, D. (2000) TFIIH is negatively regulated by cdk8-containing mediator complexes. Nature, 407, 102-106.

Bamps, S., Westerling, T., Pihlak, A., Tafforeau, L., Vandenhaute, J., Makela, T.P. and Hermand, D. (2004) Mcs2 and a novel CAK subunit Pmh1 associate with Skp1 in fission yeast. Biochem. Biophys. Res. Commun. 325, 1424-1432.

Buck, V., Russell, P. and Millar, J.B. (1995) Identification of a cdkactivating kinase in fission yeast. EMBO J. 14, 6173-6183.

Cismowski, M.J., Laff, G.M., Solomon, M.J. and Reed, S.L. (1995) KIN28 encodes a C-terminal domain kinase that controls mRNA transcription in Saccharomyces cerevisiae but lacks cyclindependent kinase-activating kinase (CAK) activity. Mol. Cell Biol. 15, 2983-2992.

Damagnez, V., Makela, T.P. and Cottarel, G. (1995) Schizosaccharomyces pombe Mop1-Mcs2 is related to mammalian CAK. EMBO J. 14, 6164-6172.

Devault, A., Martinez, A.M., Fesquet, D., Labbe, J.C., Morin, N., Tassan, J.P., Nigg, E.A., Cavadore, J.C. and Doree, M. (1995) MAT1 ('menage a trois') a new RING finger protein subunit stabilizing cyclin $\mathrm{H}$-cdk7 complexes in starfish and Xenopus CAK. EMBO J. 14, 5027-5036.

Espinoza, F.H., Farrell, A., Erdjument-Bromage, H., Tempst, P. and Morgan, D.O. (1996) A cyclin-dependent kinase-activating kinase (CAK) in budding yeast unrelated to vertebrate CAK. Science, $\mathbf{2 7 3}$ 1714-1717.

Featherstone, C. and Russell, P. (1991) Fission yeast p107wee1 mitotic inhibitor is a tyrosine/serine kinase. Nature, 349, 808-811.

Feaver, W.J., Henry, N.L., Wang, Z., Wu, X., Svejstrup, J.Q., Bushnell, D.A., Friedberg, E.C. and Kornberg, R.D. (1997) Genes for Tfb2, Tfb3, and Tfb4 subunits of yeast transcription/repair factor IIH. Homology to human cyclin-dependent kinase activating kinase and IIH subunits. J. Biol. Chem. 272, 1931919327.

Feaver, W.J., Svejstrup, J.Q., Henry, N.L. and Kornberg, R. (1994) Relationship of CDK-activating kinase and RNA polymerase II CTD kinase TFIIH/TFIIK. Cell, 79, 1103-1109.

Ferrando, A., Farras, R., Jasik, J., Schell, J. and Koncz, C. (2000) Intron-tagged epitope: a tool for facile detection and purification of proteins expressed in Agrobacterium-transformed plant cells. Plant J. 22, 553-560.

Ferrando, A., Koncz-Kalman, Z., Farras, R., Tiburcio, A., Schell, J. and Koncz, C. (2001) Detection of in vivo protein interactions between Snf1-related kinase subunits with intron-tagged epitopelabelling in plants cells. Nucleic Acids Res. 29, 3685-3693.

Fisher, R.P. and Morgan, D.O. (1994) A novel cyclin associates with M015/CDK7 to form the CDK-activating kinase. Cell, 78, 713-724.

Glab, N., Labidi, B., Oin, L.X., Trehin, C., Bergounioux, C. and Meijer, L. (1994) Olomoucine, an inhibitor of the cdc2/cdk2 kinases activity, blocks plant cells at the $\mathrm{G} 1$ to $\mathrm{S}$ and $\mathrm{G} 2$ to $\mathrm{M}$ cell cycle transitions. FEBS Lett. 353, 207-211.

Gould, K.L. and Nurse, P. (1989) Tyrosine phosphorylation of the fission yeast $\mathrm{cdc} 2^{+}$protein kinase regulates entry into mitosis. Nature, 342, 39-45.

Hata, S. (1991) cDNA cloning of a novel cdc2 ${ }^{+} / C D C 28-$ related protein kinase from rice. FEBS Lett. 279, 149-152.

Hemerly, A.S., de Almeida Engler, J., Bergounioux, C., Van Montagu, M., Engler, G., Inzé, D. and Ferreira, P. (1995) Dominant negative mutants of the $\mathrm{Cdc} 2$ kinase uncouple cell division from iterative plant development. EMBO J. 14, 3925-3936.

Ito, M., Iwase, M., Kodama, H., Lavisse, P., Komamine, A., Nishihama, R., Machida, Y. and Watanabe, A. (1998) A novel cis-acting element in promoters of plant B-type cyclin genes activates $\mathrm{M}$ phase-specific transcription. Plant Cell, 10, 331-341.

Jordan, P., Cunha, C. and Carmo-Fonseca, M. (1997) The cdk7-cyclin H-MAT1 complex associated with TFIIH is localized in coiled bodies. Mol. Biol. Cell 8, 1207-1217.

Joubès, J., Chevalier, C., Dudits, D., Heberle-Bors, E., Inze, D., Umeda, M. and Renaudi, J.P. (2000) CDK-related protein kinases in plants. Plant Mol. Biol. 43, 607-620.

Kaldis, P. (1999) The cdk-activating kinase (CAK): from yeast to mammals. Cell Mol. Life Sci. 55, 284-296.

Kaldis, P., Sutton, A. and Solomon, M.J. (1996) The Cdk-activating kinase (CAK) from budding yeast. Cell, 86, 553-564.

Kaldis, P., Pitluk, Z.W., Bany, I.A., Enke, D.A., Wagner, M., Winter, E. and Solomon, M.J. (1998) Localization and regulation of the cdkactivating kinase (Cak1p) from budding yeast. J. Cell Sci. 111, 3585-3596.

Kimmelman, J., Kaldis, P., Hengartner, C.J., Laff, G.M., Koh, S.S., Young, R.A. and Solomon, M.J. (1999) Activating phosphorylation of the Kin28p subunit of yeast TFIIH by Cak1p. Mol. Cell Biol. 19, 4774-4787.

Krempler, A., Kartarius, S., Gunther, J. and Montenarh, M. (2005) Cyclin $\mathrm{H}$ is targeted to the nucleus by $\mathrm{C}$-terminal nuclear localization sequences. Cell Mol. Life Sci. 62, 1379-1387.

Kumagai, A. and Dunphy, W.G. (1991) The cdc25 protein contains an intrinsic phosphatase activity. Cell, 67, 189-196.

Labbé, J.C., Martinez, A.M., Fesquet, D., Capony, J.P., Darbon, J.M., Derancourt, J., Devault, A., Morin, N., Cavadore, J.C. and Doree, M. (1994) p40 $0^{\mathrm{MO} 15}$ associates with a p36 subunit and requires both nuclear translocation and Thr176 phosphorylation to generate cdk-activating kinase activity in Xenopus oocytes. EMBO J. 13, 5155-5164. 
Liu, Y., Kung, C., Fishburn, J., Ansari, A.Z., Shokat, K.M. and Hahn, S. (2004) Two cyclin-dependent kinases promote RNA polymerase II transcription and formation of the scaffold complex. Mol. Cell Biol. 24, 1721-1735.

Lundgren, K., Walworth, N., Booher, R., Dembski, M., Kirschner, M. and Beach, D. (1991) mik1 and wee1 cooperate in the inhibitory tyrosine phosphorylation of cdc2. Cell, 64, 1111-1122.

Magyar, M.T., Miskolczi, P., Deak, M. et al. (1997) Cell cycle phase specificity of putative cyclin-dependent kinase variants in synchronized alfalfa cells. Plant Cell, 9, 223-235.

Mäkelä, T.P., Tassan, J.P., Nigg, E.A., Frutiger, S., Hughes, G.J. and Weinberg, R.A. (1994) A cyclin associated with the CDK-activating kinase MO15. Nature, 371, 254-257.

Menges, M., de Jager, S.M., Gruissem, W. and Murray, J.A.H. (2005) Global analysis of the core cell cycle regulators of Arabidopsis identifies novel genes, reveals multiple and highly specific profiles of expression and provides a coherent model for plant cell cycle control. Plant J. 41, 546-566.

Morgan, D.O. (1997) Cyclin-dependent kinases: engines, clocks, and microprocessors. Annu. Rev. Cell Dev. Biol. 13, 261-291.

Nagata, T., Nemoto, Y. and Hasezawa, S. (1992) Tobacco BY2 cell line as the 'HeLa' cell line in the cell biology of higher plants. Int. Rev. Cytol. 132, 1-30.

Nurse, P. (1990) Universal control mechanism regulating onset of M-phase. Nature, 344, 503-508.

Parker, L.L. and Piwnica-Worms, H. (1992) Inactivation of the p34 ${ }^{\text {cdc2 }}$-cyclin B complex by the human WEE1 tyrosine kinase. Science, 257, 1955-1957.

Parker, L.L., Atherton-Fessler, S. and Piwnica-Worms, H. (1992) $\mathrm{p} 107^{\text {wee } 1}$ is a dual-specificity kinase that phosphorylates $\mathrm{p} 34^{\mathrm{cdc} 2}$ on tyrosine 15. Proc. Natl Acad. Sci. USA, 89, 2917-2921.

Poon, R.Y., Yamashita, K., Adamczewski, J.P., Hunt, T. and Shuttleworth, J. (1993) The cdc2-related protein $\mathrm{p} 40^{\mathrm{MO} 15}$ is the catalytic subunit of a protein kinase that can activate $\mathrm{p} 33^{\mathrm{cdk} 2}$ and p34 ${ }^{\text {cdc2 }}$. EMBO J. 12, 3123-3132.

Ríos, G., Lossow, A., Hertel, B. et al. (2002) Rapid identification of Arabidopsis insertion mutants by non-radioactive detection of TDNA tagged genes. Plant J. 32, 243-253.

Russell, P. and Nurse, P. (1987) Negative regulation of mitosis by wee $1^{+}$, a gene encoding a protein kinase homolog. Cell, 49, 559567.

Schneider, E., Kartarius, S., Schuster, N. and Montenarh, M. (2002) The cyclin $\mathrm{H} / \mathrm{cdk} 7 /$ Mat1 kinase activity is regulated by CK2 phosphorylation of cyclin H. Oncogene, 21, 5031-5037.

Schwartz, B.E., Larochelle, S., Suter, B. and Lis, J.T. (2003) Cdk7 is required for full activation of Drosophila heat shock genes and RNA polymerase II phosphorylation in vivo. Mol. Cell Biol. 23 6876-6886.

Serizawa, H., Makela, T.P., Conaway, J.W., Conaway, R.C., Weinberg, R.A. and Young, R.A. (1995) Association of Cdk-activating kinase subunits with transcription factor TFIIH. Nature, 374, 280 282.

Shiekhattar, R., Mermelstein, F., Fisher, R.P., Drapkin, R., Dynlacht, B., Wessling, H.C., Morgan, D.O. and Reinberg, D. (1995) Cdk- activating kinase complex is a component of human transcription factor TFIIH. Nature, 374, 283-287.

Shimotohno, A., Matsubayashi, S., Yamaguchi, M., Uchimiya, H. and Umeda, M. (2003) Differential phosphorylation activities of CDK-activating kinases in Arabidopsis thaliana. FEBS Lett. 534, 69-74.

Shimotohno, A., Umeda-Hara, C., Bisova, K., Uchimiya, H. and Umeda, M. (2004) The plant-specific kinase CDKF;1 is involved in activating phosphorylation of cyclin-dependent kinase-activating kinases in Arabidopsis. Plant Cell, 16, 2954-2966.

Sorrell, D.A., Marchbank, A., McMahon, K., Dickinson, J.R., Rogers, H.J. and Francis, D. (2002) A WEE1 homologue from Arabidopsis thaliana. Planta, 215, 518-522.

Sun, Y., Dilkes, B.P., Zhang, C., Dante, R.A., Carneiro, N.P., Lowe, K.S., Jung, R., Gordon-Kamm, W.J. and Larkins, B.A. (1999) Characterization of maize (Zea mays L.) Wee1 and its activity in developing endosperm. Proc. Natl Acad. Sci. USA, 96, 41804185.

Tassan, J.P., Jaquenoud, M., Fry, A.M., Frutiger, S., Hughes, G.J. and Nigg, E.A. (1995) In vitro assembly of a functional human CDK7-cyclin $\mathrm{H}$ complex requires MAT1, a novel $36 \mathrm{kDa}$ RING finger protein. EMBO J. 14, 5608-5617.

Thuret, J.Y., Valay, J.G., Faye, G. and Mann, C. (1996) Civ1 (CAK in vivo), a novel Cdk-activating kinase. Cell, 86, 565-576.

Tsakraklides, V. and Solomon, M.J. (2002) Comparison of Cak1plike cyclin-dependent kinase-activating kinases. J. Biol. Chem. 277, 33482-33489.

Umeda, M. (2002) CDK-activating kinases in higher plants in CDKactivating kinase (CAK). In CDK-activating Kinase (CAK) (Kaldis, P., ed). Georgetown, TX: Landes Bioscience, pp. 55-64.

Umeda, M., Bhalerao, R.P., Schell, J., Uchimiya, H. and Koncz, C. (1998) A distinct cyclin-dependent kinase-activating kinase of Arabidopsis thaliana. Proc. Natl Acad. Sci. USA, 95, 50215026.

Umeda, M., Shimotohno, A. and Yamaguchi, M. (2005) Control of cell division and transcription by cyclin-dependent kinase-activating kinases in plants. Plant Cell Physiol. 46, 1437-1442.

Vandepoele, K., Raes, J., De Veylder, L., Rouze, P., Rombauts, S. and Inze, D. (2002) Genome-wide analysis of core cell cycle genes in Arabidopsis. Plant Cell, 14, 903-916.

Vitaly, V.K. (2000) Rapid and reliable protein extraction from yeast. Yeast, 16, 857-860.

Yamaguchi, M., Umeda, M. and Uchimiya, H. (1998) A rice homolog of Cdk7/MO15 phosphorylates both cyclin-dependent protein kinases and the carboxy-terminal domain of RNA polymerase II. Plant J. 16, 613-619.

Yamaguchi, M., Fabian, T., Sauter, M., Bhalerao, R.P., Schrader, J., Sandberg, G., Umeda, M. and Uchimiya, H. (2000) Activation of CDK-activating kinase is dependent on interaction with $\mathrm{H}$-type cyclins in plants. Plant J. 24, 11-20. Erratum in: Plant J. (2001) 25, 473.

Zuo, J., Niu, Q.W. and Chua, N.H. (2000) An estrogen receptor-based transactivator XVE mediates highly inducible gene expression in transgenic plants. Plant J. 24, 265-273.

Arabidopsis seed stock: MPI8258, SALK_120536 